\title{
Urinary Micrornas as Noninvasive Biomarkers for Acetaminophen- Induced Liver Injury
}

\author{
Xi Yang ${ }^{1}$, Zhiguang $\mathrm{Li}^{2}$, Zhenqiang Su${ }^{3}$, Kelly Davis ${ }^{4}$, Tao Chen ${ }^{2}$, Donna L Mendrick ${ }^{1}$ and William Salminen ${ }^{1 *}$ \\ ${ }^{1}$ Division of Systems Biology, Food and Drug Administration, USA \\ ${ }^{2}$ Division of Genetic and Molecular Toxicology, Food and Drug Administration, USA \\ ${ }^{3} \mathrm{Z}-\mathrm{Tech}$, an ICF International Company \\ ${ }^{4}$ Toxicologic Pathology Associates, National Center for Toxicological Research, Food and Drug Administration, 3900 NCTR Road, Jefferson, AR 72079, USA
}

\begin{abstract}
Several recent studies measured elevated levels of circulating plasma microRNAs (miRNAs) after toxicant-induced liver injury, most likely due to leakage from damaged hepatocytes. miRNAs have also been detected in urine with some of them being derived from organs outside of the urinary system, opening up their potential use as noninvasive biomarkers of disease or injury. Despite this potential, changes in urine miRNA profiles have not been investigated as biomarkers for drug-induced liver injury. In this study, urine miRNA profiles were assessed from rats treated with a single oral dose of acetaminophen (APAP: 100 or $1250 \mathrm{mg} / \mathrm{kg}$ ). The low dose did not cause any clinical pathology or histopathological changes indicative of liver injury. In contrast, the high dose increased clinical pathology and histopathological indices of liver injury at 24 hours; however, there was a high inter-animal variability in these endpoints. No evidence of kidney injury was noted at either APAP dose. Urinary miRNA levels correlated best with the degree of liver centrilobular glycogen depletion. In contrast to the high inter-animal variability noted for serum alanine and aspartate aminotransferases and centrilobular liver necrosis, urinary miRNA levels were consistently elevated in all high dose animals. Alterations in the urinary miRNA profiles were also noted in the low dose animals, albeit fewer in number. These results suggest that specific miRNAs in the urine could reflect the severity of APAP-induced liver injury and therefore have the potential to be used as noninvasive preclinical and clinical biomarkers.
\end{abstract}

\section{Keywords: MicroRNAs; Acetaminophen; Biomarkers;} Hepatotoxicity; DILI

Abbreviations: miRNA: microRNA; DILI: Drug Induced Liver Injury; APAP: Acetaminophen; NAPQI: $N$-acetyl- $p$ benzoquinoneimine; ALT: Alanine Aminotransferase; AST: Aspartate Aminotransferase; HCA: Hierarchical Clustering; PCA: Principal Component Analysis; GAPDH: Glyceraldehyde 3-Phosphate Dehydrogenase; nt: Nucleotide

\section{Introduction}

Drug induced liver injury (DILI) is a predominant reason for cessation of drug testing in clinical trials, restrictions on drug use, and the withdrawal of approved drugs [1]. Existing biomarkers of liver injury (e.g., serum alanine and aspartate aminotransferases [ALT and AST] and bilirubin) provide reasonable indicators of damage or altered function [2,3]; however, they involve invasive blood draws. It would be ideal to have a urinary biomarker of liver injury since samples could be easily collected at repeated intervals, enhancing patient compliance. Ideally, the urinary biomarker would also provide a more specific, sensitive and consistent indicator of liver injury than existing biomarkers. This study was conducted to determine if microRNAs (miRNAs), which are released from injured liver, pass through the kidney into the urine, and can subsequently serve as biomarkers of DILI.

miRNAs are small, $\sim 22$ nucleotide long, non-coding RNAs that are involved with regulating gene expression [4,5]. Functionally, miRNAs suppress gene expression by increasing mRNA degradation and decreasing protein synthesis through partial sequence complementation with their targeting mRNA. It has also been implicated in gene expression activation by up-regulation of target mRNA translation [6]. In the most current release of miRBase (http://www.mirbase.org; Release 16), there are 408 miRNAs identified in the rat. Despite the identification of a large number of miRNAs, the biological targets of many miRNAs remain elusive since this is a relatively new field. As with mRNA expression, miRNA has been reported to be dysregulated in cancer patients compared to healthy control individuals showing that miRNA may be useful biomarkers of disease or injury.

In contrast to mRNA, miRNA is much more stable and has been found in intact form in various extracellular body fluids such as plasma and urine [7-9]. Due to this stability, miRNAs have the potential to be used as noninvasive biomarkers. Further aiding their use as noninvasive urine biomarkers is the fact that the characterization and quantification processes of miRNAs are more specific and efficient than many protein biomarkers [8]. In this study, we tested the utility of urinary miRNA for detecting DILI and compared it to traditional indicators of hepatic injury.

Acetaminophen (APAP) is a widely used over-the-counter analgesic that is the leading cause of drug-induced acute liver failure in the US $[2,10]$. An APAP-induced model of DILI in rats was selected for this study since this is a well established model of liver injury that exhibits effects similar to those observed in humans. In addition, APAP has been shown to result in increased plasma miRNA levels in a similar mouse model of hepatotoxicity [11]. At low dosages, APAP is

*Corresponding author: William Salminen, Division of Systems Biology, National Center for Toxicological Research, Food and Drug Administration, 3900 NCTR Road, Jefferson, AR 72079, USA, Tel: 1-870-543-7852; E-mail:William.Salminen@ fda.hhs.gov

Received March 02, 2011; Accepted April 06, 2011; Published April 08, 2011

Citation: Yang X, Li Z, Su Z, Davis K, Chen T, et al. (2011) Urinary Micrornas as Noninvasive Biomarkers for Acetaminophen-Induced Liver Injury. J Postgenom Drug Biomark Develop 1:101. doi:10.4172/2153-0769.1000101

Copyright: (c) 2011 Yang X, et al. This is an open-access article distributed under the terms of the Creative Commons Attribution License, which permits unrestricted use, distribution, and reproduction in any medium, provided the original author and source are credited. 
extensively metabolized in the liver by glucuronidation and sulfation pathways followed by elimination via urine [12]. When administered at high dosages, the major metabolic pathways become saturated and the excess APAP is oxidized by microsomal cytochrome P450 enzymes, primarily CYP2E1, CYP1A2 and CYP3A4, forming a toxic metabolite, $\mathrm{N}$-acetyl-p-benzoquinoneimine (NAPQI). NAPQI is detoxified by conjugation with glutathione (GSH); however, once GSH stores become depleted, NAPQI can react with other macromolecules causing cellular damage, ultimately leading to liver necrosis, leakage of hepatocellular contents into the blood, and potentially liver failure [13].

In this investigation, we hypothesized that urinary miRNA patterns would reflect the biological status of the liver after APAP exposure and could serve as biomarkers of injury. To this end, we collected urine from control rats and those administered a low $(100 \mathrm{mg} / \mathrm{kg})$ or high $(1250 \mathrm{mg} / \mathrm{kg})$ dose of APAP followed by miRNA analysis using low density PCR arrays. Using these methods, we illustrate that the aberrant pattern of urinary miRNAs can be used as noninvasive biomarkers of APAP-induced liver injury.

\section{Materials and Methods}

\section{Chemicals}

APAP and methylcellulose were purchased from Sigma-Aldrich (St. Louis, MO). TRIzol reagent was purchased from Invitrogen (Carlsbad, CA).

\section{Animal care}

Six to seven week-old male Sprague-Dawley rats, provided by the US Food and Drug Administration National Center for Toxicological Research (NCTR) breeding colonies, were used for the study. Animal care was performed in accordance with the National Institutes of Health (NIH) "Guide for the Care and Use of Laboratory Animals" and was authorized by the NCTR Institutional Animal Care and Use Committee. After a seven day acclimation period, rats were assigned to experimental groups by weight averaging and housed individually in polycarbonate cages using hardwood chip bedding. During the study, room temperatures remained within $19-23^{\circ} \mathrm{C}$, and fluorescent lighting was provided on a $12 \mathrm{~h}$ on, $12 \mathrm{~h}$ off cycle. Filtered tap water was provided ad libitum and NIH-41 irradiated diet was provided ad libitum except during designated periods of fasting. Rats were fasted overnight for at least 12 hours prior to a single oral (gavage) dose of APAP (suspended in $0.5 \%$ methylcellulose) with feed given back to the animals 4 hours after dosing. There were 4 rats in the $0.5 \%$ methylcellulose control group, 4 rats in the APAP low dose $(100 \mathrm{mg} / \mathrm{kg})$ group and 7 rats in the APAP high dose $(1250 \mathrm{mg} / \mathrm{kg}$ ) group. (Since high doses of APAP can result in lethality, additional rats were included in the high dose group.) In this study, no high dose rats died prior to the planned necropsy and all high dose samples were analyzed $24 \mathrm{~h}$ after dosing.

\section{Urine sample collection and urinalysis}

Rats were acclimated to metabolism cages for approximately 24 hours prior to dosing and the start of urine collection. After dosing, the animals were returned to the metabolism cages and urine was collected into $50 \mathrm{ml}$ polypropylene containers over 24 hours. The urine containers were surrounded by a frozen salt water mixture during the collection period to prevent bacterial growth and sample degradation. This mixture quickly froze the urine in the collection tube and kept it frozen during the entire collection period. After thawing on ice, an aliquot was removed and subject to urinalysis for the following parameters: glucose, protein, bilirubin, ketones, urobilinogen, nitrites, blood, leukocytes, micro protein, urine creatinine, and urine glucose.
The remaining urine was centrifuged at $10,000 \times \mathrm{g}$ for 10 minutes at $4^{\circ} \mathrm{C}$ to pellet cellular debris and then aliquoted and frozen at $-80^{\circ} \mathrm{C}$.

\section{Necropsy, biochemical and hematological analyses and histopathology}

Rats were anesthetized with carbon dioxide, blood was withdrawn via cardiac puncture, and the rats were then euthanized by carbon dioxide asphyxiation. Blood was collected into serum separator or ethylenediaminetetraacetic acid (EDTA) tubes (BD, Franklin Lakes, $\mathrm{NJ}$ ). Blood in the serum separator tube was allowed to clot for $30-$ $60 \mathrm{~min}$ at room temperature, centrifuged at $300 \times \mathrm{g}$ for approximately 10 minutes, and the serum analyzed on an automated clinical chemistry analyzer (Alfa Wassermann ALERA, West Caldwell, NJ) for creatinine, blood urea nitrogen (BUN), ALT, AST, alkaline phosphatase (ALP), gamma glutamyl transferase (GGT), glucose, cholesterol, triglycerides, chloride, sodium, potassium, calcium, phosphorus, albumin, total protein, and total bilirubin. Blood in the EDTA tube was analyzed the same day as collection on an ABX Pentra $60 \mathrm{C}+$ analyzer (ABX, Irvine, CA) for red and white blood cell counts, hemoglobin, hematocrit, mean corpuscular hemoglobin concentration, mean corpuscular volume, platelets, and differential white blood cell count. A gross necropsy was conducted on all animals and included the examination of all major organs, body cavities, and external surfaces. The brain, heart, kidney, liver, lung, and testis were weighed. Sections of the liver and kidneys were fixed in $10 \%$ neutral buffered formalin, routinely processed, embedded in paraffin, sectioned at $5 \mu \mathrm{m}$, stained with hematoxylin and eosin, and examined by light microscopy by a boardcertified veterinary pathologist. Lesions were scored on a 5-point scale (0-normal, 1-minimal, 2-mild, 3-moderate, 4-marked). Serum ALT and AST data were analyzed using a one-way ANOVA followed by a Dunn's Test. Differences among groups were considered significant at $p<0.05$. Serum ALT and AST values were log-transformed prior to statistical analysis.

\section{RNA isolation from urine}

Total RNA from $300 \mu \mathrm{L}$ urine was extracted using the TRIzol ${ }^{\circ}$ method according to the manufacturer's protocol. Briefly, $1 \mathrm{ml}$ of TRIzol ${ }^{\circ}$ was added to $300 \mu \mathrm{l}$ of urine. The sample was mixed and incubated for $5 \mathrm{~min}$ at room temperature, followed by the addition of $200 \mu \mathrm{l}$ chloroform. After mixing vigorously for $15 \mathrm{sec}$, the sample was centrifuged at $12,000 \times \mathrm{g}$ at $4^{\circ} \mathrm{C}$ for $15 \mathrm{~min}$. The upper aqueous phase was carefully transferred to a new tube, and $500 \mu$ lisopropanol was added to precipitate the RNA. The RNA pellet was cleaned in $1 \mathrm{ml} 75 \%$ ethanol and redissolved in RNase-free water. Total RNA concentrations were quantitated on a NanoDrop spectrophotometer (NanoDrop Technologies, Wilmington, DE). Qualitative analyses were performed on each sample using the Agilent small RNA kit on the Agilent 2100 bioanalyzer (Agilent Technologies, Palo Alto, CA)

\section{miRNA measurement by PCR Arrays}

Three hundred nanograms of urine RNA were converted into cDNA using a $\mathrm{RT}^{2}$ miRNA First Strand Kit (Qiagen/SABiosciences Corporation, Frederick, MD). The percentage of RNA in the miRNA size range (10 - $40 \mathrm{nt})$ was similar among control and APAP treated groups, so similar amounts of miRNAs were converted into cDNA to eliminate possible variations. All the synthesized cDNAs were mixed with $2 \times \mathrm{RT}^{2}$ SYBR Green PCR Master Mix (Qiagen/SABiosciences) and dispersed into a 384-well Rat Genome miRNA PCR Array (MAR$3100 \mathrm{E}$, Qiagen/SABiosciences) with $10 \mu \mathrm{l} /$ well reaction volume. In order to decrease the loading variance, all the cDNA mixes were loaded by a Beckman Coulter Biomek FX Liquid Handler (Beckman Coulter 
Citation: Yang X, Li Z, Su Z, Davis K, Chen T, et al. (2011) Urinary Micrornas as Noninvasive Biomarkers for Acetaminophen-Induced Liver Injury. J Postgenom Drug Biomark Develop 1:101. doi:10.4172/2153-0769.1000101

INC., Brea, CA). The PCR array contained a panel of primer sets for 370 rat miRNAs, four blanks, four small RNAs as the internal controls, three reverse transcription controls (RTC) and three positive PCR controls (PPC). The real-time qRT-PCR was performed on a 7900 real-time PCR system (Applied Biosystems Inc., Foster, CA) with the following cycling parameters: $95^{\circ} \mathrm{C}$ for $10 \mathrm{~min}$, then 40 cycles of $95^{\circ} \mathrm{C}$ for $15 \mathrm{sec}, 60^{\circ} \mathrm{C}$ for $30 \mathrm{sec}$ and $72^{\circ} \mathrm{C}$ for $30 \mathrm{sec}$. SYBR Green fluorescence was recorded from every well during the annealing step of each cycle. The threshold cycle $(\mathrm{Ct})$ value of each sample was calculated with software SDS 2.3 (Applied Biosystems). The baseline was automatically defined by the software and the threshold line was set as 0.15 across all the plates. All the samples passed the quality controls based on the Ct values of RTC and PPC. Any Ct value less than 35 was considered as a positive call.

\section{Normalization and statistical analysis}

Relative miRNA expression levels were determined with the $\Delta$ Ct method, normalized with miR-193, miR-323*, and miR-675 and reported as $2^{-\Delta \mathrm{Ct}}$, where $\Delta \mathrm{Ct}=\mathrm{Ct}$ (miRNA of interest) $-\mathrm{Ct}$ (housekeeping). miR-193, miR-323*, and miR-675 were geometrically averaged [14] as the "housekeeping" endogenous controls since these exhibited stable levels across the control and two treated groups. Fold change was calculated as: fold change $=2^{[- \text {-mean of } \Delta C \text { t values of treated samples }- \text { mean }}$ of $\Delta$ Ct values of control samples)]. The Welch's t-test was used to calculate the $p$ value to determine whether there was a significant difference for miRNA expression between the control and the APAP high dose or low dose group for each miRNA.

\section{Global visualization methods}

Hierarchical clustering on two different datasets was used to obtain a global view of the urine miRNA profiles of each control animal compared to each APAP treated animal. All hierarchical clustering was performed using Ward's minimum variance method. In the first dataset, the control and APAP high dose groups were subjected to hierarchical clustering analysis using the 44 miRNAs found to be significantly changed in the APAP high dose group $(p<0.01$, fold change $>1.5)$. In the second dataset, the control and APAP low and high dose groups were subjected to clustering analysis using the miRNAs that were increased by low dose APAP treatment. At $p<0.01$, no miRNA changes were significant from the low dose group, so a $p$-value less than 0.05 were used to select the affected miRNAs. There were 9 miRNAs whose expression was significantly increased $(p<0.05$, fold change $>1.5$ ) by low dose APAP treatment. All nine of these miRNAs were also significantly increased in the high APAP dose group. Principal component analysis of significantly altered miRNAs from APAP high or low dose groups was conducted using ArrayTrack ${ }^{\mathrm{TM}}$ [15] (free genomic analysis software available from the USFDA NCTR at http://www.fda. gov/ScienceResearch/BioinformaticsTools/Arraytrack/default.htm). The normalized $\Delta \mathrm{Ct}$ values were used for this analysis and the analysis was performed without filtering any miRNAs.

\section{In silico functional analysis of the significant miRNAs}

The miRanda database was used for identification of potential target genes regulated by the 44 significantly altered miRNAs in the APAP high dose group (http://microrna.sanger.ac.uk/sequences/). The top $5 \%$ of the most reliably predicted target genes of the APAP high dose related miRNAs were selected according to the $p$ values given in the database. The selected target genes were then input into Ingenuity Pathway Analysis (IPA) software (Ingenuity Systems, Inc., Redwood City, CA). Ingenuity Core Analysis and Knowledge Base was used as the reference set. IPA interpreted the genes in the context of biological processes, pathways and molecular networks and defined the most relevant biological functions related to these miRNAs.

\section{Results}

\section{Clinical chemistry and histopathology endpoints of APAP- induced liver toxicity}

A single oral high dose of APAP $(1250 \mathrm{mg} / \mathrm{kg})$ produced classic

\begin{tabular}{|c|c|c|c|c|c|}
\hline & \multirow[t]{2}{*}{ Animal \# } & \multicolumn{2}{|l|}{ Activity, (IU/L) } & \multicolumn{2}{|l|}{ Histopathology } \\
\hline & & ALT & AST & Centrilobular Glycogen Depletion & Centrilobular Necrosis \\
\hline \multirow{5}{*}{ Control } & 1 & 69 & 114 & 0 & 0 \\
\hline & 2 & 65 & 90 & 0 & 0 \\
\hline & 3 & 71 & 105 & 0 & 0 \\
\hline & 4 & 65 & 83 & 0 & 0 \\
\hline & MEAN +/- SD & $67.5 \pm 3.0$ & $98.0 \pm 14.1$ & 0 & 0 \\
\hline \multirow{5}{*}{$100 \mathrm{mg} / \mathrm{kg}$ APAP } & 5 & 47 & 91 & 0 & 0 \\
\hline & 6 & 55 & 122 & 0 & 0 \\
\hline & 7 & 59 & 120 & 0 & 0 \\
\hline & 8 & 59 & 76 & 0 & 0 \\
\hline & MEAN +/- SD & $55.0 \pm 5.7$ & $102.2 \pm 22.5$ & 0 & 0 \\
\hline \multirow{8}{*}{$1250 \mathrm{mg} / \mathrm{kg}$ APAP } & 9 & 59 & 127 & 1 & 0 \\
\hline & 10 & 1523 & 7580 & 4 & 3 \\
\hline & 11 & 69 & 131 & 1 & 0 \\
\hline & 12 & 71 & 108 & 2 & 1 \\
\hline & 13 & 65 & 117 & 2 & 2 \\
\hline & 14 & 68 & 103 & 1 & 0 \\
\hline & 15 & 1364 & 12880 & 4 & 3 \\
\hline & MEAN +/- SD & $459.9 \pm 673.5^{*}$ & $3006.6 \pm 5166.3$ & $2.1 \pm 1.3$ & $1.3 \pm 1.4$ \\
\hline
\end{tabular}

Note. Rats were administered a single oral gavage dose of APAP and serum was collected $24 \mathrm{~h}$ after dosing. Histopathology lesions were scored on a 5 point scale $(0=$ normal, $1=$ minimal, $2=$ mild, $3=$ moderate, $4=$ marked).

*Statistically significant increase compared to the low dose APAP $(100 \mathrm{mg} / \mathrm{kg})$ group $(\mathrm{p} \leq 0.05)$. Histopathology scores were not statistically analyzed.

Table 1: Inter-individual variability in traditional biochemical and histopathological indices of hepatocellular injury $24 \mathrm{~h}$ after a single oral dose of APAP. 
Citation: Yang X, Li Z, Su Z, Davis K, Chen T, et al. (2011) Urinary Micrornas as Noninvasive Biomarkers for Acetaminophen-Induced Liver Injury. J Postgenom Drug Biomark Develop 1:101. doi:10.4172/2153-0769.1000101

Page 4 of 10

\begin{tabular}{|l|l|l|}
\hline miRNA ID & Ct value & S.D. \\
\hline rno-miR-323* & 23.59 & $0.31^{\#}$ \\
\hline rno-miR-92a & 24.21 & 0.53 \\
\hline rno-miR-193 & 24.32 & $0.12^{\#}$ \\
\hline rno-miR-125b-3p & 25.22 & 1.01 \\
\hline rno-miR-667 & 25.30 & 0.36 \\
\hline rno-miR-1224 & 25.52 & 1.16 \\
\hline rno-miR-431 & 26.84 & 1.14 \\
\hline rno-miR-212 & 27.06 & 0.95 \\
\hline rno-miR-770 & 27.31 & 0.96 \\
\hline rno-miR-139-3p & 27.61 & 0.48 \\
\hline rno-miR-92b & 27.63 & 1.16 \\
\hline rno-miR-675 & 27.65 & $0.20^{\#}$ \\
\hline rno-miR-140* & 27.69 & 0.54 \\
\hline rno-miR-125b-5p & 27.73 & 1.72 \\
\hline rno-miR-26a & 27.95 & 1.52 \\
\hline
\end{tabular}

Note. The urinary miRNAs in the control group are listed in descending order with the highest levels on the top. \#: "House-keeping" internal control miRNAs are marked.

Table 2: The top 15 most abundant miRNAs in rat urine.

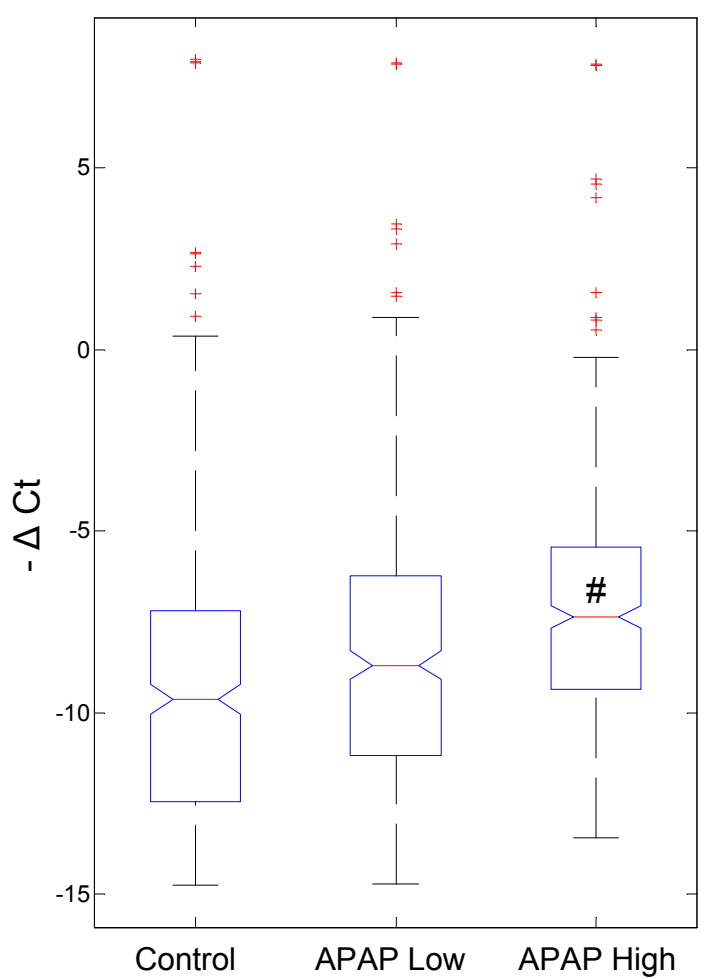

Figure 1: Box plot of global urinary miRNA levels in control, $100 \mathrm{mg} / \mathrm{kg}$ APAP (Low) and $1250 \mathrm{mg} / \mathrm{kg}$ APAP (High) groups. Global miRNA (370 rat miRNAs on the PCR array) level from each group was reported as average $-\Delta$ Ct normalized with miR-193, miR-323*, and miR-675. \#: APAP high dose group showed an increase in the global miRNA levels $(p<0.01$, t-test).

signs of liver toxicity consisting of increased serum ALT and AST and centrilobular liver glycogen depletion and necrosis (Table 1). There was a high inter-animal variability in the ALT and AST responses with some animals exhibiting very high levels and others having levels similar to controls. The histopathology produced a more consistent response with all high dose animals exhibiting centrilobular glycogen depletion and 4 of 7 animals exhibiting some degree of centrilobular necrosis, a classic lesion of APAP hepatocellular injury $[13,16]$. The centrilobular glycogen

depletion appeared to be a sensitive indicator of APAP-induced injury, is consistent with previous studies in mice and rats [17,18], and may be directly influenced by the APAP reactive metabolite [18]. Alternatively, the centrilobular glycogen may have been simply due to the decreased feed consumption noted in the high dose animals (data not shown).

The low dose of APAP $(100 \mathrm{mg} / \mathrm{kg})$ did not produce any classical signs of liver toxicity. APAP has been shown to induce kidney lesions in some strains of rats (e.g., Fischer 344 ) at doses that cause liver toxicity [19]. The Sprague Dawley strain, the strain used in this study, is resistant

\begin{tabular}{|c|c|c|c|}
\hline miRNA ID & Control & $100 \mathrm{mg} / \mathrm{kg}$ APAP & $1250 \mathrm{mg} / \mathrm{kg}$ APAP \\
\hline rno-miR-376a & $0.2 \pm 0.1$ & $1.1 \pm 0.5$ & $6.0 \pm 2.1$ \\
\hline rno-miR-130a & $0.5 \pm 0.1$ & $3.1 \pm 1.6$ & $8.5 \pm 2.5$ \\
\hline rno-miR-674-3p & $0.5 \pm 0.2$ & $2.6 \pm 0.5$ & $7.8 \pm 2.2$ \\
\hline rno-miR-463 & $0.6 \pm 0.3$ & $1.5 \pm 0.6$ & $4.0 \pm 1.0$ \\
\hline rno-miR-185 & $0.6 \pm 0.3$ & $0.7 \pm 0.3^{\#}$ & $6.7 \pm 1.7$ \\
\hline rno-miR-17-5p & $0.6 \pm 0.2$ & $4.2 \pm 1.7$ & $11.5 \pm 3.4$ \\
\hline rno-miR-485 & $0.6 \pm 0.5$ & $0.5 \pm 0.1^{\#}$ & $3.7 \pm 0.5$ \\
\hline rno-miR-152 & $0.7 \pm 0.4$ & $1.0 \pm 0.5^{\#}$ & $4.9 \pm 1.1$ \\
\hline rno-miR-425 & $0.8 \pm 0.4$ & $1.3 \pm 0.5$ & $4.4+0.9$ \\
\hline rno-miR-449a & $0.8 \pm 0.4$ & $3.5 \pm 1.9$ & $13.0 \pm 5.2$ \\
\hline rno-miR-181c & $0.8 \pm 0.4$ & $1.2 \pm 0.6^{\#}$ & $6.0 \pm 1.1$ \\
\hline rno-miR-327 & $0.8 \pm 0.4$ & $0.7 \pm 0.2^{\#}$ & $3.8 \pm 0.9$ \\
\hline rno-miR-151* & $0.9 \pm 0.4$ & $1.7 \pm 0.6$ & $13.1 \pm 5.1$ \\
\hline rno-miR-380 & $0.9 \pm 0.1$ & $1.4 \pm 0.8$ & $3.5 \pm 0.7$ \\
\hline rno-miR-664 & $0.9 \pm 0.6$ & $2.7 \pm 0.6$ & $7.2 \pm 1.4$ \\
\hline rno-miR-343 & $1.0 \pm 0.7$ & $2.5 \pm 1.2$ & $7.6 \pm 1.7$ \\
\hline rno-miR-379 & $1.0 \pm 0.2$ & $3.0 \pm 1.2$ & $3.9 \pm 1.0$ \\
\hline rno-miR-135a* & $1.1 \pm 0.5$ & $2.7 \pm 0.6$ & $5.2 \pm 0.7$ \\
\hline rno-miR-124* & $1.1 \pm 0.4$ & $3.7 \pm 1.2$ & $7.4 \pm 2.0$ \\
\hline rno-miR-344-5p & $1.2 \pm 0.7$ & $2.3 \pm 1.0$ & $7.9 \pm 2.6$ \\
\hline rno-miR-423 & $1.2 \pm 0.4$ & $1.4 \pm 0.4^{\#}$ & $10.2 \pm 3.4$ \\
\hline rno-miR-291a-5p & $1.3 \pm 0.5$ & $6.6 \pm 1.7$ & $20.4 \pm 5.0$ \\
\hline rno-miR-30d* & $1.4 \pm 0.6$ & $4.3 \pm 1.1$ & $10.3 \pm 3.2$ \\
\hline rno-miR-342-3p & $1.5 \pm 0.6$ & $4.9 \pm 1.3$ & $25.7 \pm 10.1$ \\
\hline rno-miR-542-5p & $1.6 \pm 1.0$ & $5.4 \pm 2.0$ & $10.7 \pm 2.0$ \\
\hline rno-miR-330* & $2.0 \pm 0.8$ & $2.6 \pm 0.4^{\#}$ & $18.3 \pm 6.4$ \\
\hline rno-miR-222 & $2.0 \pm 0.7$ & $10.1 \pm 5.3$ & $14.5 \pm 4.0$ \\
\hline rno-miR-134 & $2.1 \pm 0.8$ & $5.0 \pm 1.9$ & $20.2 \pm 4.9$ \\
\hline rno-miR-328 & $2.1 \pm 0.7$ & $2.9 \pm 1.0^{\#}$ & $18.6 \pm 4.0$ \\
\hline rno-miR-433 & $2.3 \pm 0.8$ & $4.4 \pm 2.1$ & $38.6 \pm 9.9$ \\
\hline rno-miR-221 & $2.5 \pm 0.5$ & $4.5 \pm 1.2$ & $8.8 \pm 2.1$ \\
\hline rno-miR-203 & $2.6 \pm 1.0$ & $12.9 \pm 3.4$ & $32.8 \pm 12.8$ \\
\hline rno-miR-434 & $2.6 \pm 0.9$ & $4.9 \pm 1.3$ & $18.9 \pm 5.3$ \\
\hline rno-miR-298 & $2.7 \pm 1.3$ & $4.3 \pm 1.2$ & $15.4 \pm 2.8$ \\
\hline rno-miR-20b-3p & $3.2 \pm 1.4$ & $5.2 \pm 1.1$ & $27.6 \pm 10.6$ \\
\hline rno-miR-99b* & $4.1 \pm 2.2$ & $10.8 \pm 5.1$ & $41.9 \pm 10.4$ \\
\hline rno-miR-34c* & $5.2 \pm 0.8$ & $7.2 \pm 1.4^{\#}$ & $44.5 \pm 15.8$ \\
\hline rno-miR-484 & $5.3 \pm 1.2$ & $6.1 \pm 0.7^{\#}$ & $31.7 \pm 11.0$ \\
\hline rno-miR-760-3p & $5.5 \pm 1.6$ & $16.1 \pm 2.9$ & $24.8 \pm 3.0$ \\
\hline rno-miR-191 & $5.6 \pm 0.8$ & $7.9 \pm 1.7^{\#}$ & $36.4 \pm 8.8$ \\
\hline rno-miR-489 & $13.8 \pm 2.5$ & $40.7 \pm 9.1$ & $54.4 \pm 10.9$ \\
\hline rno-let-7b* & $14.7 \pm 4.3$ & $23.8 \pm 5.5$ & $74.0 \pm 20.4$ \\
\hline rno-miR-296 & $16.7 \pm 5.7$ & $37.1 \pm 7.6$ & $93.4 \pm 22.6$ \\
\hline rno-miR-92b & $114.8 \pm 43.5$ & $213.1 \pm 51.8$ & $575.2 \pm 135.3$ \\
\hline
\end{tabular}

Note. The relative miRNA levels were calculated by $2^{-\Delta C t}$, normalized to three housekeeping miRNAs (miR-193, miR-323*, and miR-675), and presented as mean + SEM, $p<0.01$. \#: Fold change less than 1.5 compared between APAP low dose and the control.

Table 3: The 44 miRNAs that were significantly increased in the $1250 \mathrm{mg} / \mathrm{kg}$ APAP high dose urine samples. 
Citation: Yang X, Li Z, Su Z, Davis K, Chen T, et al. (2011) Urinary Micrornas as Noninvasive Biomarkers for Acetaminophen-Induced Liver Injury. J Postgenom Drug Biomark Develop 1:101. doi:10.4172/2153-0769.1000101

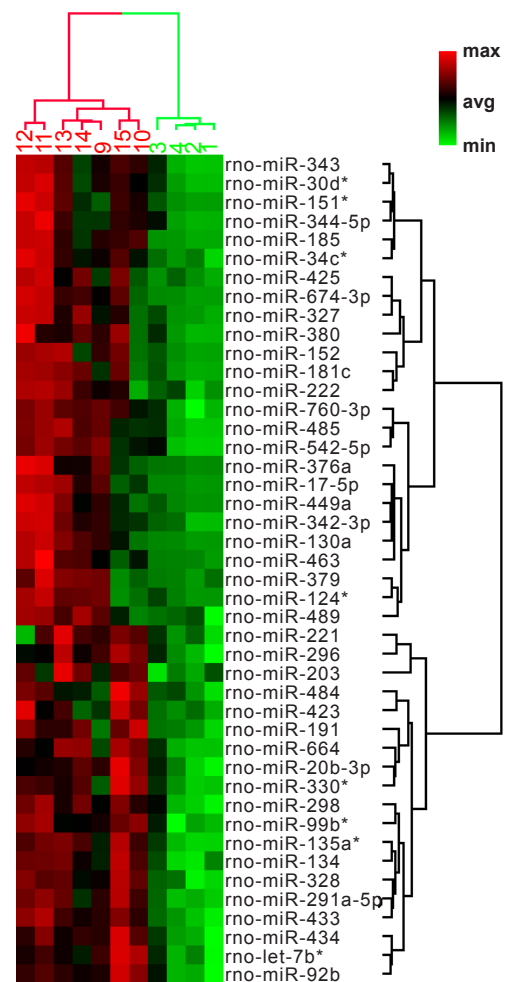

Figure 2: Hierarchical clustering analysis of 44 differentially affected urinary miRNAs. The Welch's t-test $(p<0.01)$ was used to identify the significantly changed ( $\triangle \mathrm{Ct}$ value) urinary miRNAs at $24 \mathrm{~h}$ after APAP dosing. The figure shows the clustering analysis of the 44 miRNAs that showed a $>1.5$-fold change between control (green animal numbers) and $1250 \mathrm{mg} / \mathrm{kg}$ APAP high dose group (red animal numbers). Relative miRNA concentration was reported as $2^{-\Delta C t}$ and the progressively brighter shades of red indicate higher miRNA levels; whereas, the green indicates lower levels. The identities of miRNAs are listed on the right and the animal numbers are on the top in color.

to APAP-induced nephrotoxicity and no evidence of kidney injury was observed based on clinical chemistry (e.g., BUN and creatinine) and histopathology indices (data not shown).

\section{Data normalization for urine miRNA}

We adapted the $\mathrm{RT}^{2}$ miRNA PCR Array from Qiagen/SABiosciences to profile the expression of the 370 most abundantly expressed and best characterized miRNA sequences in the rat genome as annotated by the Sanger miRBase Release 14.

Of the 230 miRNAs positively detected in urine samples from untreated rats (Ct values less than 35 from at least three samples), the top 15 miRNAs with the highest levels are shown in Table 2. Three of these (miR-193, miR-323*, and miR-675) were also present at relatively high and invariant levels in control and treated samples. Normalization using the mean of multiple internal control genes is currently the preferred method for qRT-PCR data. The software geNorm was used to validate that these three miRNAs were the most reliable internal controls [14]. All of them were found to be the most stable miRNAs with stability values (M) less than 1.5 . Therefore, miR-193, miR-323*, and miR-675 were used as internal control "housekeeping" miRNAs for normalization purposes. Other normalization procedures typically used for tissues (e.g., global normalization or normalization against noncoding small nuclear RNAs such as U6) were not suitable for normalization in urine.

\section{Significant elevation of miRNA global mean levels in the urine of APAP high dose rats}

It has been reported that there was a significant increase of total RNA in mouse plasma $24 \mathrm{~h}$ after a hepatotoxic dose of APAP [11]. From the rat urine, the amount of total RNA from control and APAP treated rats were similar, ranging from $3 \mu \mathrm{g}$ to $10 \mu \mathrm{g}$ per $300 \mu$ l urine. Based on the estimation from the Agilent 2100 Bioanalyzer, the percentage of RNA in the miRNA size range (10 - $40 \mathrm{nt}$ ) was similar among control, APAP low dose, and APAP high dose groups. Interestingly, the miRNA global expression levels were significantly ( $p<0.01$ by t-test) increased in the APAP high dose animals (Figure 1), even though equal amounts of urine total RNA (300 ng) from each rat were used in the PCR arrays. The box plot of the urinary miRNA levels also showed an upward trend with increasing APAP dosage.

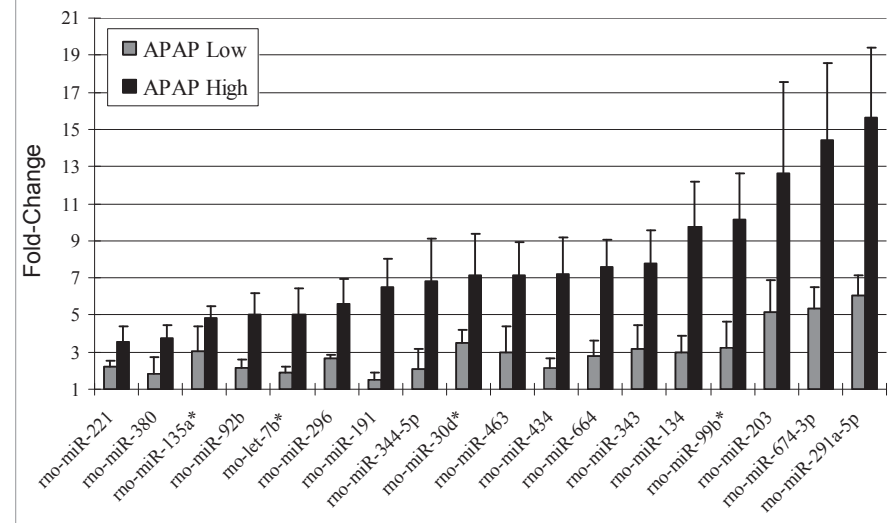

Figure 3: Dose-dependent fold changes of selected miRNAs altered by treatment with APAP at $24 \mathrm{~h}$. The Welch's t-test $(p<0.01)$ was used to identify the significantly changed ( $\triangle$ Ct value) urinary miRNAs at $24 \mathrm{~h}$ after $1250 \mathrm{mg} /$ $\mathrm{kg}$ APAP dosing. Fold change was compared between APAP treated $(100 \mathrm{mg} /$ $\mathrm{kg}$ or $1250 \mathrm{mg} / \mathrm{kg}$ ) to the control group, calculated as fold change $=2^{\text {[-(mean of } \Delta C}$ values of treated samples - mean of $\triangle \mathrm{Ct}$ values of control samples)]. The values are the average of at least 4 independent samples and the standard errors are shown as plus error bars.

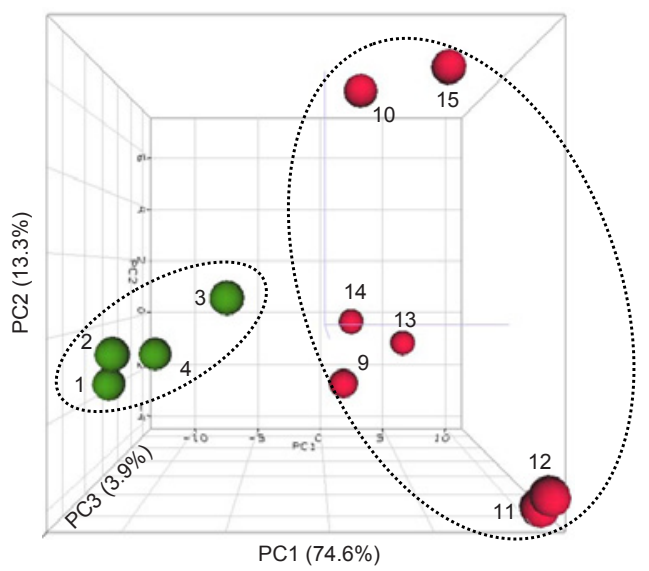

Figure 4: Principal component analysis of 44 differentially affected urinary miRNAs. Samples from the controls are indicated in green and samples from the $1250 \mathrm{mg} / \mathrm{kg}$ APAP high dose group are in red. The animal numbers are adjacent to the markers. The Welch's t-test $(p<0.01)$ was used to identify the significantly changed ( $\triangle \mathrm{Ct}$ value) urinary miRNAs at $24 \mathrm{~h}$ after APAP dosing. The control and APAP high dose groups are separated along the first principal component $(74.6 \%)$. 
A.

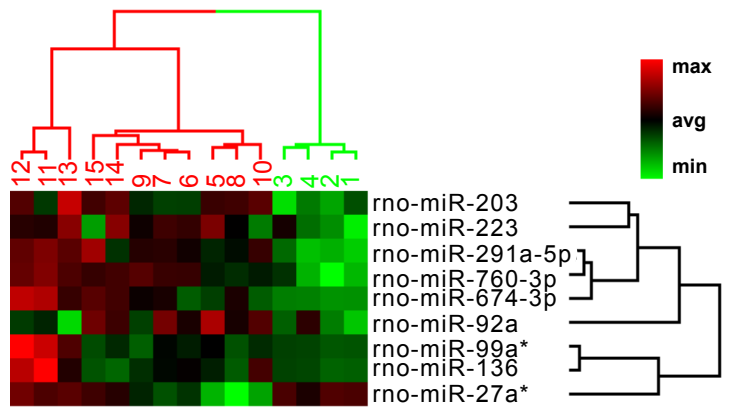

Figure 5: HCA and PCA properties based on the 9 differentially affected was used to identify the significantly changed ( $\triangle$ Ct value) urinary miRNAs at $24 \mathrm{~h}$ after a $100 \mathrm{mg} / \mathrm{kg} \mathrm{APAP}$ low dose. A. The 15 samples (4 from control [green numbers 1 - 4], 4 from APAP low dose [red numbers $5-8$ ], and 7 from APAP high dose [red numbers $9-15$ ]) can be grouped into 2 major clusters based on the 9 significant miRNAs identified from the APAP low dose group. B. Control group is separated from APAP low dose and high dose groups along the second principal component (38.2

$\%)$. Fifteen animals were included in the analysis, and the animal numbers are adjacent to the markers.

B.

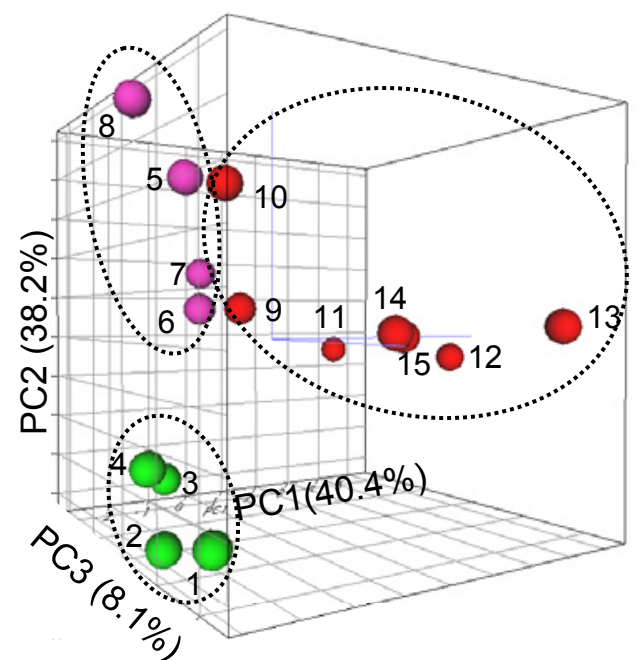

APAP low dose urine samples. The Welch's t-test $(p<0.05)$ APAP exposure induced differential patterns of miRNA in urine

Unsupervised hierarchical clustering analysis of the 230 positively detected $(\mathrm{Ct}<35)$ miRNAs revealed two major clusters: most (3 of 4 rats) in control group vs. APAP high dose group (Figure S1). The Welch's t-test identified 44 miRNAs that were significantly different $(p$ $<0.01$, fold change $>1.5$ ) between the control and APAP high dose groups. Clustering of the samples was performed a second time with 11 urine miRNA samples (4 control and 7 APAP high dose) using the 44 significantly changed miRNAs selected by the Welch's t-test. The resulting dendrogram was again divided into two clearly separated branches: a first branch containing the control urine samples $(n=4)$ and a second containing the seven APAP treated urine samples (Figure 2 ), emphasizing that the miRNA profile of the APAP high dose group was dramatically different from the control. Table 3 summarizes the significant changes in the $44 \mathrm{miRNAs}$ detected in urine samples from the APAP low and high dose groups. miR-193, miR-323*, and miR-675 were used as "housekeeping" miRNAs and the miRNA relative expression levels were calculated by $2^{-\Delta C t}$. In general, for these significantly changed miRNAs selected from the APAP high dose group, most showed a dosedependent increase after APAP exposure (Table 3 and Figure 3). Some miRNAs, for example miR-185, were considered as negative (Ct value greater than 35) in the control and low dose groups; however, they were positively detected in the high dose group (Table 3 ).

Principal component analysis (PCA) of the control and APAP high dose samples using the 44 miRNAs revealed that miRNA PCR array data from each rat could be clearly divided into two groups. Figure 4 shows that the APAP-high dose samples separated from control along PC1, which accounts for $74.6 \%$ of the variability in the data. Within the APAP high dose group, animals showed a much higher level of variation than the control, which may be caused by larger inter-individual responses to the APAP treatment, as was observed with the classical endpoints of liver toxicity. Specifically, the PCA shown in Figure 4 indicates that rat numbers 10 and 15 were situated in dimensional space very far away from the control group. These two animals had the most severe liver injury as assessed by serum ALT and AST levels and severity of liver necrosis.

Changes in the pattern of urinary miRNA levels were also observed in the APAP low dose group, which were exposed to a subtoxic dose of only $100 \mathrm{mg} / \mathrm{kg}$. Applying a $p$ value less than 0.05 as the cutoff, Welch's t-test analysis of the APAP low dose group yielded nine significant miRNAs that showed a fold change $>1.5$. These nine miRNAs were also significantly changed in the APAP high dose group. Hierarchical clustering analysis was performed on 15 urine samples (4 control, 4 APAP low dose, and 7 APAP high dose), with the 9 miRNAs. The resulting dendrogram was again divided into two clearly separated branches: one branch containing the 4 control urine samples and the other branch containing the 11 APAP-treated urine samples (Figure $5 \mathrm{~A}$ ). To better evaluate the dose dependency of miRNA levels, PCA was generated using the same 9 miRNAs. Figure 5B shows the 3D PCA plot for control, APAP low dose and APAP high dose. There is a clear separation of the two dosed groups from the control group. The control group animals formed a tight cluster in the plot apart from the treated rats. Further, the high dose group can be clearly separated from the low dose group along PC1.

These clustering and PCA results indicated that the miRNA patterns occurring in the urine were influenced by the APAP treatment in a dose-dependent manner, and that, among the APAP high dose group, response variations may also be distinguished by urinary miRNA levels.

\section{Urine miRNAs show promise as biomarkers for liver injury}

The serum ALT level, a widely used biomarker for liver function, was determined in all serum samples collected in the experiment. The urine miRNA-291a-5p, which was significantly increased in both low dose and high dose APAP groups, was plotted together with serum ALT levels to illustrate the similarity in changes between these two different types of biomarkers (Figure 6 and Figure S2). The variations of ALT levels in the high dose APAP-treated group were very large and, interestingly, some animals in this group did not have an increase over control levels (Table 1). In contrast, miRNA-291a-5p levels from each 


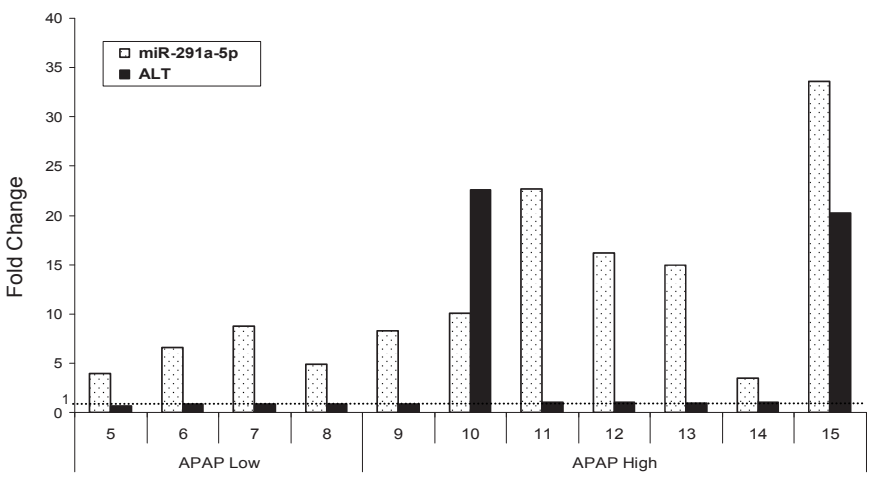

Figure 6: miRNA biomarker is more sensitive and consistent than ALT for liver injury. miRNA-291a-5p fold change was compared between APAP treated $(100 \mathrm{mg} / \mathrm{kg}$ or $1250 \mathrm{mg} / \mathrm{kg})$ and the control group, calculated as fold change $=2^{[-(\Delta C \text { t value of treated sample - mean of } \Delta C \text { t values of control samples })]}$. ALT fold change was compared to the mean ALT value in the control group.

$-\log (p$-value $)$

$0.00 .10 .2 \quad 0.3 \quad 0.40 .5 \quad 0.60 .70 .80 .90 .91 .11 .21 .31 .41 .51 .61 .71 .81 .92 .0$

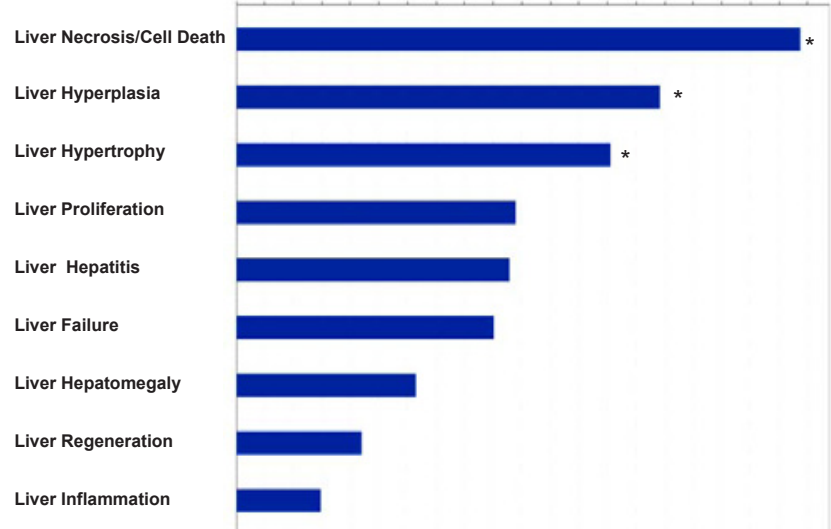

Figure 7: Liver functions altered by APAP treatment predicted by the 44 miRNAs. The target genes of the 44 significantly increased miRNAs were computationally predicted by miRanda algorithm. The top $5 \%$ predicted genes were utilized for functional analysis using Ingenuity Pathway Analysis system. Liver functions are listed according to the $p$ values. ${ }^{*}, p<$ 0.05 .

APAP high dose treated rat showed increased urinary concentrations (Figure 6). This trend was noted for other miRNAs too (data not shown). These data suggest that urine miRNA might be a more consistent biomarker of APAP-induced injury than the current serum ALT and AST tests. In addition, a smaller set of miRNAs were increased at the low dose of APAP $(100 \mathrm{mg} / \mathrm{kg})$, which did not cause any classic signs of liver toxicity, indicating that some miRNAs may also be more sensitive than existing biomarkers of liver injury (Figure 3).

The 44 significant miRNAs' target genes (Top 5\%, 346 genes) were input into Ingenuity Pathway Analysis (IPA) software and the related functions and pathways were determined and are provided in Figure 7. Liver necrosis/cell death was the highest ranked biological function affected by the APAP high does treatment. Networks including cell death, cellular assembly and cell signaling were potentially affected by miRNA species that had increased levels in the urine.

\section{Discussion}

Serum ALT and AST are widely used biochemical markers for hepatocellular injury, however, both enzymes can increase from other types of organ injury such as increased ALT from skeletal muscle injury [2,9]. In addition, serum ALT and AST activities do not always correlate well wth liver changes as increased serum ALT activity absent of any histopathological changes and vice versa can occur [20-23] This was observed in our study where some high dose animals had centrilobular glycogen depletion and/or centrilobular necrosis without a concomitant increase in serum ALT or AST (e.g., see animals \#12 and \#13 in Table 1). Other biochemical markers of liver injury (e.g., serum bilirubin, alkaline phosphatase, gamma glutamyl transferase) are more specific for cholestatic injury or complete liver failure [24] and some of them also suffer from non-specific increases from extrahepatic organ damage, such as increased alkaline phosphatase from bone injury or remodeling [25]. Therefore, it would be beneficial to develop new biomarkers of hepatocellular injury that are both more specific and consistent than existing biochemical markers of injury. Identifying urinary biomarkers specific for liver injury would be an added benefit since it would avoid the use of invasive blood draws. In addition, urine samples would enhance patient compliance since it would involve an inexpensive patient-controlled procedure that can be done more routinely.

This study was conducted to determine if urinary miRNAs, which are released from injured liver into the systemic circulation, pass through the kidney into the urine, and can subsequently serve as a biomarker of DILI. In contrast to extracellular full length DNA and mRNA, miRNA is very stable in extracellular fluids $[8,26]$. Some RNA-binding proteins have been reported to protect extracellular miRNAs from degradation and some miRNAs are present in high concentrations in body fluids [8]. This unique stability and enrichment may aid in the utility of miRNAs as biomarkers in body fluids that can be obtained non-invasively, such as urine. Further aiding their use as non-invasive biomarkers is the fact that the characterization and quantification process of miRNAs are more specific and efficient than many protein biomarkers [27]. Developing new protein based biomarkers is limited by the complexity of protein composition, post-translational modification of proteins, and the low abundance of many proteins in body fluids. Biopsies of the liver can be performed for histopathological evaluation; however, they are invasive procedures and involve higher risks for the patient. Thus, it is recognized that novel diagnostic and prognostic indicators that can be obtained from noninvasive biomaterials are required [1].

Previous studies have detected transrenal urinary DNA and RNA and specific assays are being developed to monitor the transrenal genetic biomarkers and better assess the health status of humans $[28,29]$ and see Trovagene.com website). Therefore, it is not surprising that not only were miRNAs detected in rat urine but they also exhibited differential patterns after APAP-induced liver injury. Various factors such as viral hepatitis, fatty liver, liver fibrosis, hepatocellular carcinoma, and DILI have been shown to produce abnormal patterns of miRNA expression in the liver [5]. Extending this observation, recent studies suggested that the detection of miRNAs in plasma is a promising approach to identify novel biomarkers for DILI. Wang et al [11] showed higher levels of mmu-miR-122, mmu-miR-192, and several other miRNAs in plasma after APAP-induced liver injury in mice. It seems that the increase of miRNAs in body fluids is due to the release of cellular miRNAs or pre-packaged miRNA exosomes, reflecting the APAP-induced cellular damage in the liver $[11,26]$. Despite these reports, there is no published study on the levels of urinary miRNA after DILI.

We investigated the potential utility of urine miRNAs as noninvasive biomarkers for the diagnosis of DILI using an APAP overdosed rat model. We observed consistent increases of 44 urine miRNAs in 
animals treated with a hepatotoxic dose of APAP $(1250 \mathrm{mg} / \mathrm{kg})$, a classical liver toxicant, over a $24 \mathrm{~h}$ collection period. Such increases were observed even in APAP high dose rats that did not present with ALT or AST increases (Figure 6). The urinary levels of some miRNAs were also increased in the low dose APAP group $(100 \mathrm{mg} / \mathrm{kg})$, which produced no classical signs of hepatotoxicity. Previous studies have demonstrated that subtoxic APAP exposure caused significant changes in liver mRNA expression profiles and mitochondrial damage in centrilobular hepatocytes as assessed with electron microscopy [30,31]. Whether the changes in urinary miRNA are in fact sensitive biomarkers for cellular injury or are specifically related to APAP toxicity is presently unknown. It is interesting to note that for most of these 44 urinary miRNAs identified from the APAP high dose group, there was a dose-dependent increase in the APAP low dose group (Table 3 and Figure 3). These findings highlight that urinary miRNAs may be both more sensitive and more consistent biomarkers of liver injury compared to serum ALT and AST. Several studies have looked at the organ specificity of various miRNAs $[11,27,32]$. None of the 44 urine miRNAs detected in this study were cross-referenced to previously identified liver-specific miRNAs. However, these previous studies used different species and/or strains and analytical platforms. Therefore, it is difficult to conclude if the detected urinary miRNAs are specific for liver injury. Additional work will be required to further determine the specificity of the findings since it is possible that other compounds, regardless of their hepatotoxicity potential, may also cause a similar increase in urinary miRNAs.

MiR-122 has been shown to be highly liver specific with expression levels in the liver being several thousand times higher than in other tissues. Several recent reports showed that miR-122 increased in plasma after liver injury and exhibited a diagnostic sensitivity superior to ALT $[9,11,27]$. However, in our study, we detected miR-122 in urine but there was no significant change of urine miR-122 after APAP treatment. One possible reason for the discrepancy between miR-122 levels in the plasma versus urine could be due to the different types of body fluids. It has been reported that the composition of miRNA species in the urine does not show substantial overlap with the plasma [8]. In fact, the comparison of miRNAs between different body fluids showed that plasma and urine shared the fewest number of commonly detectable miRNAs. Given this, it is likely that miRNAs have different rates of transrenal passage into the urine, thereby, limiting the specific miRNAs that can be detected. Another possibility is that miR-122 was not increased in the plasma of the treated rats and additional plasma miRNA analysis would be required to definitively determine this. In the study by Wang et al., miR-130a was increased in the plasma of APAPtreated mice [11]. We observed a similar increase in miR-130a in the rat urine samples.

It is important to analyze the functions of the 44 miRNAs that were significantly increased in the urine of this study together because each miRNA can regulate many different target genes. Here we used the miRanda algorithm, which was developed by the Sanger Institute and is widely used in miRNA studies [33,34], to predict the target genes. Mechanistically, it is not clear how these miRNAs and the downstream mRNA targets were dysregulated in response to the APAP-induced liver injury. It is, however, interesting to note that the genes targeted by these 44 miRNAs are highly associated with liver damage such as liver necrosis, liver hypertrophy, liver failure, liver proliferation and liver regeneration. These findings are consistent with previous studies demonstrating that APAP causes many physiological changes and subcellular effects in the liver. It is likely that APAP-induced hepatocellular injury causes up-regulation of the miRNA in the liver with subsequent release into the plasma and transrenal transfer to the urine. Therefore, increased urinary miRNAs may be an indirect indicator of the biological pathways affected in the liver.

In some strains of rats, APAP can cause kidney injury at doses that cause liver injury $[12,19]$. If nephrotoxicity occurred in this study, it is conceivable that the altered urinary miRNAs were simply due to the nephrotoxicity and not the liver injury. The Sprague-Dawley strain of rat used in this study is resistant to APAP nephrotoxicity and we did not observe any signs of nephrotoxicity based on histopathological and clinical chemistry endpoints. Given the potential sensitivity of genomics analysis, particularly PCR, it is possible that subclinical kidney injury occurred and this resulted in the increased urinary miRNAs. The pattern of urinary miRNA levels in our APAP high dose group was dramatically different than the pattern of urinary miRNA levels after ischemic kidney damage in rats (personal communication with Dr. Vishal Vaidya of Brigham and Women's Hospital, Boston, MA) indicating that kidney injury was most likely not the cause of the increased urinary miRNAs in the current study. In addition, miRNAs that have been associated with kidney diseases were not altered in either of our APAP dose groups [35].

The selection of an appropriate internal control under different pathological conditions is still an unsolved issue in tissue miRNA microarray and RT-PCR assays since even traditional housekeeping genes, such as GAPDH, can be altered under some conditions [36]. Data normalization is an even bigger challenge for urine miRNA analysis since urine is a dynamically changing fluid and it is likely that the concentration of various macromolecules also changes. We found several miRNAs that had stable levels across animals and treatment groups with miR-193, miR-323*, and miR-675 having the highest and most consistent levels in the urine. These three urine "housekeeping" miRNAs also showed stable expression in tumorigen- or carcinogentreated mice livers [34,37]. It has been shown that using the geometric mean of multiple internal control genes leads to a more accurate normalization then conventional use of a single gene [14]. Therefore, these three miRNAs were selected for normalization of the current dataset. The utility of these miRNAs for normalization under different pathological conditions will only be determined after further study. Several noncoding small nuclear RNAs (such as U6, U87, 4.5S-V1 and Y1) have been suggested for sample-to-sample normalization in cellular and tissue miRNA analysis. However, the levels of these were highly variable in the urine samples and were not considered appropriate for normalization. Global normalization procedures, which are normally used for extracellular miRNA measurement, are not appropriate for this study because the global means of the $\mathrm{Ct}$ values were increased after the APAP treatment (Figure 1). The increase in global means of the Ct values is not surprising since it is likely that elevated levels of miRNAs are released from injured liver into the blood and this in turn results in higher transrenal miRNA levels in the urine. We also tested the possibility of adding urinary creatinine as a normalization correction factor [38]; however, the sample variations within the group were largely increased after adding urinary creatinine (data not shown). A recent clinical study also reported that normalization to urinary creatinine may be misleading for urinary biomarkers of kidney injury [39]. Therefore, urinary miRNA levels were calculated based on the normalization to the geometric mean of the three urine "housekeeping" miRNAs (miR-193, miR-323*, and miR-675) identified in our study, reproduced in another urine miRNA study using a model of ischemic kidney injury (personal communication with Dr. Vishal Vaidya), and validated by analyzing publicly available miRNA data $[34,37]$.

In conclusion, the urinary level of 44 miRNAs was increased after a hepatotoxic dose of APAP and a smaller number of miRNAs were 
Citation: Yang X, Li Z, Su Z, Davis K, Chen T, et al. (2011) Urinary Micrornas as Noninvasive Biomarkers for Acetaminophen-Induced Liver Injury. J Postgenom Drug Biomark Develop 1:101. doi:10.4172/2153-0769.1000101

increased after a lower subtoxic dose of APAP. These results highlight the potential utility of urinary miRNA as sensitive and more consistent biomarkers of DILI when compared to traditional endpoints such as serum ALT and AST. Additional work is underway to determine if these findings can be replicated for other models of hepatic injury. If confirmed, urinary miRNA analysis might offer an early, noninvasive diagnosis for DILI in both pre-clinical and clinical settings.

\section{Disclaimer}

This article is not an official guidance or policy statement of the US Food and Drug Administration (FDA). No official support or endorsement by the US FDA is intended or should be inferred.

\section{Acknowledgments}

We thank William S. Branham for his technical expertise. We are grateful to the Bionetics Corporation for providing support for the in-life studies. Drs. X Yang and Zhiguang Li are supported by the Research Participation Program at the National Center for Toxicological Research administered by the Oak Ridge Institute for Science and Education through an interagency agreement between the US Department of Energy and the US Food and Drug Administration.

\section{References}

1. Fannin RD, Russo M, O'Connell TM, Gerrish K, Winnike JH, et al. (2010) Acetaminophen dosing of humans results in blood transcriptome and metabolome changes consistent with impaired oxidative phosphorylation. Hepatology 51: 227-236.

2. Larson AM, Polson J, Fontana RJ, Davern TJ, Lalani E, et al. (2005) Acetaminophen-induced acute liver failure: Results of a United States multicenter, prospective study. Hepatology 42:1364-1372

3. Shi Q, Hong H, Senior J, Tong W (2010) Biomarkers for drug-induced liver injury. Expert Rev Gastroenterol Hepatol 4: 225-234.

4. Carrington JC, Ambros V (2003) Role of MicroRNAs in Plant and Animal Development. Science 301: 336-338.

5. Bala S, Marcos M, Szabo G (2009) Emerging role of microRNAs in liver diseases. World J Gastroenterol 15: 5633-5640.

6. Ma F, Liu X, Li D, Wang P, Li N, et al. (2010) MicroRNA-466I Upregulates IL10 Expression in TLR-Triggered Macrophages by Antagonizing RNA-Binding Protein Tristetraprolin-Mediated IL-10 mRNA Degradation. J Immunol 184: 6053-6059.

7. Bail S, Swerdel M, Liu H, Jiao X, Goff LA, et al. (2010) Differential regulation of microRNA stability. RNA 16: 1032-1039.

8. Weber JA, Baxter DH, Zhang S, Huang DY, How Huang K, et al. (2010) The MicroRNA Spectrum in 12 Body Fluids. Clin Chem 56: 1733-1741.

9. Zhang $Y$, Jia $Y$, Zheng R, Guo Y, Wang Y, et al. (2010) Plasma MicroRNA-122 as a Biomarker for Viral-, Alcohol-, and Chemical-Related Hepatic Diseases. Clin Chem 56: 1830-1838.

10. Khandelwal N, James LP, Sanders C, Larson AM, Lee WM, et al. (2011) Unrecognized acetaminophen toxicity as a cause of indeterminate acute liver failure. Hepatology 53: 567-576

11. Wang K, Zhang S, Marzolf B, Troisch P, Brightman A, et al. (2009) Circulating microRNAs, potential biomarkers for drug-induced liver injury. Proc Natl Acad Sci U S A 106: 4402 - 4407.

12. Bessems JG, Vermeulen NP (2001) Paracetamol (Acetaminophen)-Induced Toxicity: Molecular and Biochemical Mechanisms, Analogues and Protective Approaches. Crit Rev Toxicol 31: 55-138.

13. Hinson JA, Roberts DW, James LP (2010) Mechanisms of AcetaminophenInduced Liver Necrosis. Handb Exp Pharmacol 2010: 369-405.

14. Vandesompele J, De Preter K, Pattyn F, Poppe B, Van Roy N, et al. (2002) Accurate normalization of real-time quantitative RT-PCR data by geometric averaging of multiple internal control genes. Genome Biol 3: 0034.

15. Fang H, Harris S, Su Z, Chen M, Qian F, et al. (2009) ArrayTrack: An FDA and Public Genomic Tool Methods Mol Biol 563: 379 - 398.

16. Salminen WF, Voellmy R, Roberts SM (1998) Effect of N-Acetylcysteine on
Heat Shock Protein Induction by Acetaminophen in Mouse Liver. J Pharmacol Exp Ther 286: 519-524

17. Davis DC, Potter WZ, Jollow DJ, Mitchell JR (1974) Species differences in hepatic glutathione depletion, covalent binding and hepatic necrosis after acetaminophen. Life Sci 14: 2099-2109.

18. Hinson JA, Mays JB, Cameron AM (1983) Acetaminophen-induced hepatic glycogen depletion and hyperglycemia in mice. Biochem Pharmacol 32: 19791988.

19. Newton JF, Bailie MB, Hook JB (1983) Acetaminophen nephrotoxicity in the rat. Renal metabolic activation in vitro. Toxicol Appl Pharmacol 70: 433-444.

20. Solter PF (2005) Clinical Pathology Approaches to Hepatic Injury. Toxicol Pathol 33: 9-16.

21. Thulin P, Rafter I, Stockling K, Tomkiewicz C, Norjavaara E, et al. (2008) PPARalpha regulates the hepatotoxic biomarker alanine aminotransferase (ALT1) gene expression in human hepatocytes. Toxicol Appl Pharmacol 231: $1-9$

22. Ennulat D, Magid-Slav M, Rehm S, Tatsuoka KS (2010) Diagnostic Performance of Traditional Hepatobiliary Biomarkers of Drug-Induced Liver Injury in the Rat Toxicol Sci 116: 397-412.

23. Ozer JS, Chetty R, Kenna G, Koppiker N, Karamjeet P, et al. (2010) Recommendations to qualify biomarker candidates of drug-induced liver injury. Biomark Med 4: 475-483.

24. Ramaiah SK (2007) A toxicologist guide to the diagnostic interpretation of hepatic biochemical parameters. Food Chem Toxicol 45: 1551-1557.

25. Nakagawa $H$, Kamimura $M$, Takahara $K$, Hashidate $H$, Kawaguchi $A$, et al. (2006) Changes in total alkaline phosphatase level after hip fracture: comparison between femoral neck and trochanter fractures. J Orthop Sci 11 : 135-139.

26. Wang K, Zhang S, Weber J, Baxter D, Galas DJ (2010) Export of microRNAs and microRNA-protective protein by mammalian cells. Nucleic Acids Res 38 7248-7259.

27. Laterza OF, Lim L, Garrett-Engele PW, Vlasakova K, Muniappa N, et al. (2009) Plasma MicroRNAs as Sensitive and Specific Biomarkers of Tissue Injury. Clin Chem 55: 1977-1983.

28. Umansky SR, Tomei LD (2006) Transrenal DNA testing: progress and perspectives. Expert Rev Mol Diagn 6: 153-163.

29. Melkonyan HS, Feaver WJ, Meyer E, Scheinker V, Shekhtman EM, et al. (2008) Transrenal Nucleic Acids: From Proof of Principle to Clinical Tests. Ann N Y Acad Sci 1137: 73-81.

30. Heinloth AN, Irwin RD, Boorman GA, Nettesheim P, Fannin RD, et al. (2004) Gene expression profiling of rat livers reveals indicators of potential adverse effects. Toxicol Sci 80:193-202.

31. Bushel PR, Heinloth AN, Li J, Huang L, Chou JW, et al. (2007) Blood gene expression signatures predict exposure levels. Proc Natl Acad Sci U S A 104: 18211-18216.

32. Linsen S, de Wit E, de Bruijn E, Cuppen E (2010) Small RNA expression and strain specificity in the rat. BMC Genomics 11: 249

33. Griffiths-Jones S, Saini HK, van Dongen S, Enright AJ (2008) miRBase: tools for microRNA genomics. Nucleic Acids Research 36: D154-D158.

34. Li Z, Branham W, Dial S, Wang Y, Guo L, et al. (2010) Genomic analysis of microRNA time-course expression in liver of mice treated with genotoxic carcinogen N-ethyl-N-nitrosourea. BMC Genomics 11: 609

35. Aguilar AL, Piskol R, Beitzinger M, Zhu JY, Kruspe D, et al. (2010) The small RNA expression profile of the developing murine urinary and reproductive systems. FEBS Lett 584: 4426-4434.

36. Galiveti CR, Rozhdestvensky TS, Brosius Jr, Lehrach H, Konthur Zn (2010) Application of housekeeping npcRNAs for quantitative expression analysis of human transcriptome by real-time PCR. RNA 16: 450-461.

37. Ross JA, Blackman CF, Thai S-F, Li Z, Kohan M, et al. (2010) A potentia microRNA signature for tumorigenic conazoles in mouse liver. Mol Carcinog 49: 320-323. 
Citation: Yang X, Li Z, Su Z, Davis K, Chen T, et al. (2011) Urinary Micrornas as Noninvasive Biomarkers for Acetaminophen-Induced Liver Injury. J Postgenom Drug Biomark Develop 1:101. doi:10.4172/2153-0769.1000101

Page 10 of 10

38. Goldstein SL (2010) Urinary kidney injury biomarkers and urine creatinine normalization: a false premise or not? Kidney Int 78: 433-435.
39. Waikar SS, Sabbisetti VS, Bonventre JV (2010) Normalization of urinary biomarkers to creatinine during changes in glomerular filtration rate. Kidney Int 78: 486-494. 